\title{
PSYCHOSIS AS A MULTISYSTEM DISORDER A Global Perspective
}

Guadalupe Marinho ${ }^{1}{ }^{*}$, Diogo Almeida ${ }^{1} \delta$, Maria do Carmo $\mathrm{Cruz}^{2}{ }^{*}$

${ }^{1}$ Resident, ${ }^{2}$ Senior Consultant and Head of Psychiatric Department

*Psychiatric and Mental Health Department - Portimão Unit - Centro Hospitalar Universitário Do Algarve, EPE, Portimão, Portugal

$\delta$ Psychiatric and Mental Health Department - Hospital Prof. Doutor Fernando Fonseca, EPE, Lisboa, Portugal

\section{BACKGROUND}

People with psychotic disorders die earlier than the general population, and $60 \%$ of this relates to non-central nervous systems (CNS). Secondary effects of the illness have traditionally been blamed. However, studies of people with first-episode psychosis (FEP) have shown dysfunction in cardiometabolic, immune and endocrine systems. This suggests that psychotic disorders comprehend multiple systems at onset.

\section{OBJECTIVES}

To analyse the dysfunction

in multiple systems present at the FEP.

\section{METHODS}

A literature review was conducted by searching in PubMed database the terms: "psychosis AND dysfunction AND (immune OR cardiometabolic OR endocrine OR central nervous system)". Articles were selected with regard to their scientific relevance. The search was limited to FEP to limit secondary effects of illness.

\section{RESULTS}

- The evidence suggests that early in psychosis, alterations are present across multiple organ systems - immune, cardiometabolic and endocrine:

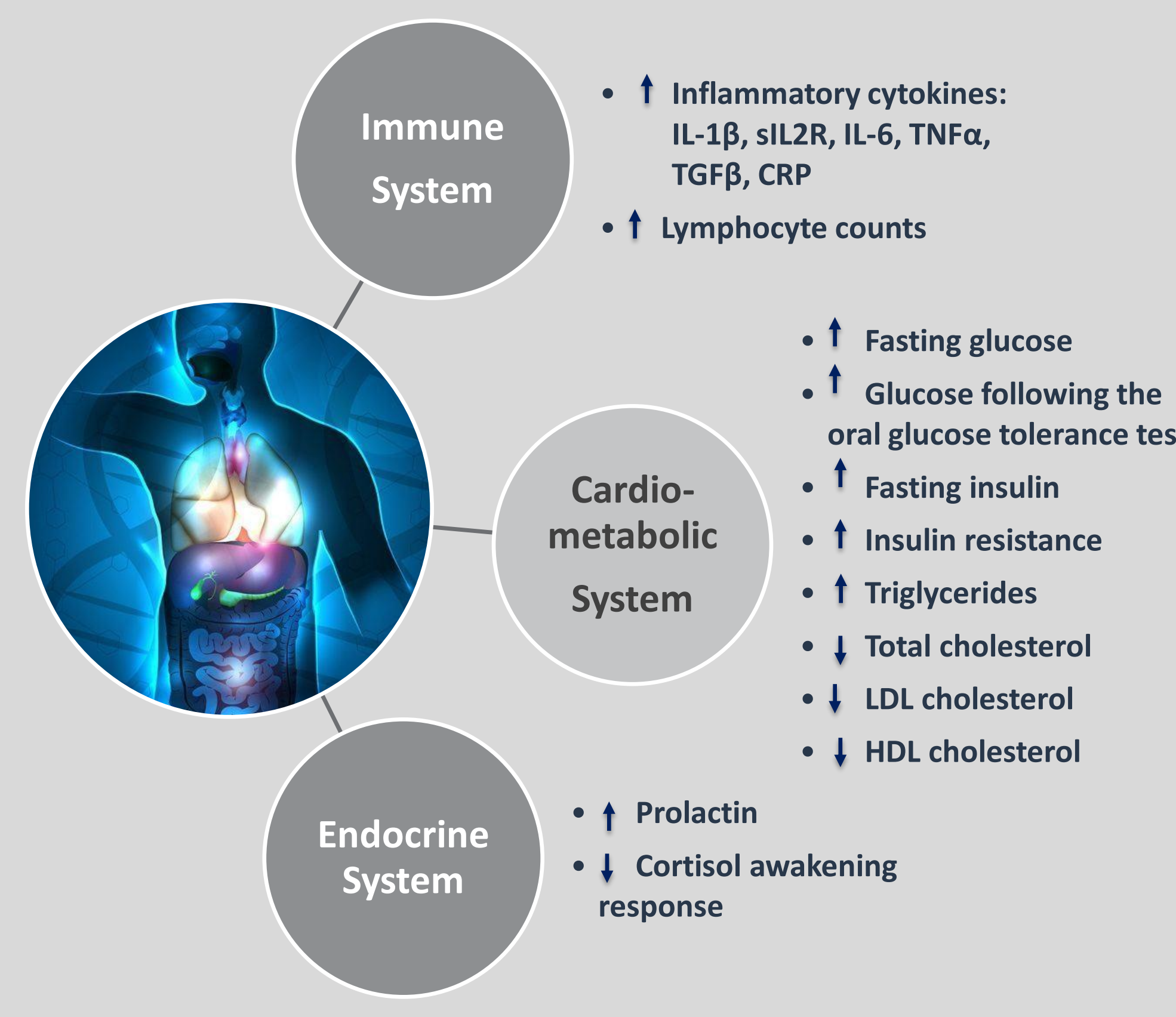

- There may be a link between some non-CNS parameters and symptom severity:

In antipsychotic naive FEP, positive symptom severity correlates indirectly with fasting glucose levels and insulin resistance;

IL-6 levels correlate directly with total psychopathology.
- Non-CNS abnormalities occur with similar effect sizes as CNS abnormalities in FEP.

- Non-CNS abnormalities may be a cause or consequence of CNS dysfunction in psychosis or an epiphenomenon. Clarification of the causal relationship is required.

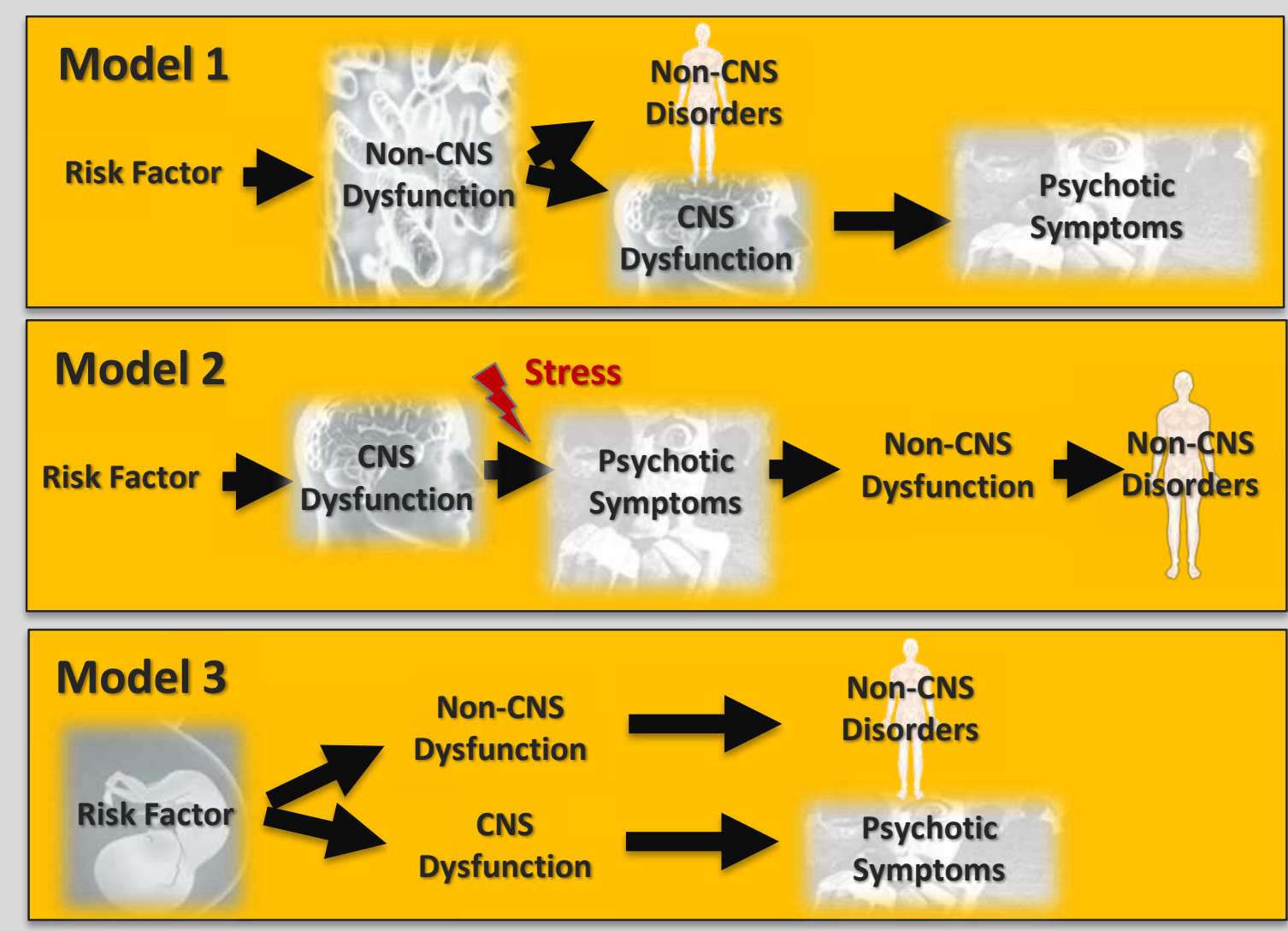

Models of the relationship between psychosis and non-CNS dysfunction. Model 1: A risk factor induces non-CNS dysfunction, which may consequently impact CNS function to increase the risk of psychosis. Model 2: A risk factor induces CNS dysfunction and thence psychotic symptoms, which may consequently trigger non-CNS dysfunction. Model 3: A shared risk factor may result in the development of psychosis and non-CNS dysfunction through independent mechanisms.

\section{CONCLUSIONS}

The evidence demonstrates that dysfunction in multiple systems is present in early psychosis and non-CNS measures are connected to symptoms and CNS changes in FEP. This suggests that psychosis should be considered a multisystem disorder. A consequence of this would possibly be that diagnostic and prognostic assessment might include assessment of non-CNS organ dysfunction. However, studies are needed to determine if addressing non-CNS alterations early decreases development of physical co-morbidity, and ultimately reduces mortality in psychotic disorders. 